\title{
COMBINATORIAL SET THEORY AND CARDINAL FUNCTION INEQUALITIES
}

\author{
R. E. HODEL
}

(Communicated by Dennis Burke)

\begin{abstract}
Three theorems of combinatorial set theory are proven. From the first we obtain the de Groot inequality $|X| \leq 2^{h L(X)}$, the Ginsburg-Woods inequality $|X| \leq 2^{e(X) \Delta(X)}$, the Erdös-Rado Partition Theorem for $n=2$, and set-theoretic versions of the Hajnal-Juhász inequalities $|X| \leq 2^{c(X) \chi(X)}$ and $|X| \leq 2^{s(X) \psi(X)}$. From the second we obtain a generalization of the Arhangel' skii inequality $|X| \leq 2^{L(X) \chi(X)}$. From the third we obtain the Charlesworth inequality $n(X) \leq p s w(X)^{L(X)}$ and a generalization of the Burke-Hodel inequality $|K(X)| \leq 2^{e(X) p s w(X)}$.
\end{abstract}

\section{INTRODUCTION}

It is well known that combinatorial set theory plays an important role in the theory of cardinal functions. Perhaps the best example of this is the use of the Erdös-Rado Partition Theorem to prove the Hajnal-Juhász inequalities $|X| \leq 2^{s(X) \psi(X)}$ and $|X| \leq 2^{c(X) \chi(X)}$. In this paper we further explore the close connection between theorems of combinatorial set theory and cardinal function inequalities.

Standard set-theoretic notation is used; $\kappa$ and $\lambda$ denote infinite cardinals; $\omega$ is the first infinite cardinal; $\alpha, \beta, \gamma, \delta$ denote ordinals; ${ }^{\kappa} A$ is the set of all functions from $\kappa$ into the set $A ;[E]^{2}=\{\{x, y\}: x, y \in E$ and $x \neq y\}$; if $\mathscr{G}$ is a collection of sets, $\operatorname{ord}(x, \mathscr{G})$ is the number of elements of $\mathscr{G}$ which contain $x$; a cover $\mathscr{S}$ of a set $E$ is separating if given distinct $x, y$ in $E$, there exists $S \in \mathscr{S}$ such that $x \in S, y \notin S$.

Cardinal function notation is also fairly standard: $n w, L, h L, c, e, p s w$, $\Delta, \chi, \psi$, and $t$ denote net weight, Lindelöf degree, hereditary Lindelöf degree, cellularity, extent, point separating weight, diagonal degree, character, pseudocharacter, and tightness, respectively.

Received by the editors November 6, 1989.

1980 Mathematics Subject Classification (1985 Revision). Primary 54A25, 04A20; Secondary 54D20.

Key words and phrases. Lindelöf degree, extent, separating cover, Erdös-Rado Partition Theorem, cardinal functions. 
The cardinal function extent is used on several occasions in this paper and so we discuss it in some detail. By definition, the extent of a topological space $X$, denoted $e(X)$, is the infinite cardinal defined by

$$
e(X)=\sup \{|D|: D \text { a closed, discrete subset of } X\}+\omega .
$$

This cardinal function has numerous characterizations, as the following lemma shows.

Lemma 1. Let $X$ be a $T_{1}$ space, let $\kappa$ be an infinite cardinal. The following are equivalent:

(1) every subset of $X$ of cardinality greater than $\kappa$ has a limit point;

(2) every closed, discrete subset of $X$ has cardinality at most $\kappa$;

(3) if $\mathscr{G}$ is an open cover of $X$ and $A \subset X$, there exists $B \subset A$ with $|B| \leq \kappa$ such that $A \subset \operatorname{st}(B, \mathscr{G})$;

(4) if $H$ is a closed subset of $X$ and $\mathscr{G}$ is an open collection in $X$ which covers $H$, there exists $B \subset H$ with $|B| \leq \kappa$ such that $H \subset \operatorname{st}(B, \mathscr{G})$;

(5) every locally finite collection of subsets of $X$ has cardinality at most $\kappa$.

To justify Lemma 1 , note that $(1) \Leftrightarrow(2)$ is obvious; the proof that $(2) \Rightarrow(3)$ (for the countable case) appears in [H2]; (3) $\Rightarrow(4)$ and (4) $\Rightarrow(1)$ are easy; $(5) \Rightarrow(1)$ is obvious, and the proof that $(1) \Rightarrow(5)$ appears in [H1].

\section{First COMBinatorial theOREM}

In the statement of Theorem I, $\mathrm{S}$ is for separating, $\mathrm{HC}$ is for hereditary cover.

Theorem I. Let $\kappa$ be an infinite cardinal, let $E$ be a set. For each $x \in E$ and each $\gamma<\kappa$ let $V(\gamma, x)$ be a subset of $E$ which contains $x$. Assume that

(S) if $x \neq y$, there exists $\gamma<\kappa$ such that $y \notin V(\gamma, x)$;

(HC) for each $\gamma<\kappa$ and each $A \subset E$, there exists $B \subset A$ with $|B| \leq \kappa$ such that $A \subset \bigcup_{x \in B} V(\gamma, x)$.

Then $|E| \leq 2^{\kappa}$.

Proof. Construct a sequence $\left\{E_{\alpha}: 0 \leq \alpha<\kappa^{+}\right\}$of subsets of $E$ such that these two conditions hold for all $\alpha<\kappa^{+}$:

(1) $\left|E_{\alpha}\right| \leq 2^{\kappa}$;

(2) if $\left\{B_{\gamma}: \gamma<\kappa\right\}$ is a collection of at most $\kappa$ subsets of $\bigcup_{\beta<\alpha} E_{\beta}$ with $\left|B_{\gamma}\right| \leq \kappa$ for each $\gamma<\kappa$, and $W \neq E$, where $W=\bigcup_{\gamma<\kappa}\left(\bigcup_{x \in B_{\gamma}} V(\gamma, x)\right)$, then $E_{\alpha}-W \neq \varnothing$.

Let $L=\bigcup_{\alpha<\kappa^{+}} E_{\alpha}$; clearly $|L| \leq 2^{\kappa}$, and so the proof is complete if $L=E$. Suppose not, and let $y \in E-L$. For each $\gamma<\kappa$ let $A_{\gamma}=\{x: x \in L$ and $y \notin V(\gamma, x)\}$. By $(\mathrm{S}), L=\bigcup_{\gamma<\kappa} A_{\gamma}$. By (HC), for each $\gamma<\kappa$ there exists $B_{\gamma} \subset A_{\gamma}$ with $\left|B_{\gamma}\right| \leq \kappa$ such that $A_{\gamma} \subset \bigcup_{x \in B_{\gamma}} V(\gamma, x)$. Let $W=$ $\bigcup_{\gamma<\kappa}\left(\bigcup_{x \in B_{\gamma}} V(\gamma, x)\right)$, and note that $L \subset W$ and $y \notin W$. Choose $\alpha$ so large that $\bigcup_{\gamma<\kappa} B_{\gamma} \subset \bigcup_{\beta<\alpha} E_{\beta}$. By (2), $E_{\alpha}-W \neq \varnothing$. This contradicts $L \subset W$. 
Corollary 1 (de Groot). For $X \in T_{2},|X| \leq 2^{h L(X)}$.

Proof. Let $\kappa=h L(X)$. Now $\psi(X) \leq h L(X)$ for $X$ Hausdorff, hence for each point $x \in X$ there is a collection $\{V(\gamma, x): \gamma<\kappa\}$ of open neighborhoods of $x$ such that $\bigcap_{\gamma<\kappa} V(\gamma, x)=\{x\}$. Clearly (S) and (HC) of Theorem I hold so $|X| \leq 2^{k}$.

Corollary 2 (Ginsburg-Woods). For $X \in T_{1},|X| \leq 2^{e(X) \Delta(X)}$.

Proof. Let $\kappa=e(X) \Delta(X)$. Since $\Delta(X) \leq \kappa$, there is a sequence $\left\{\mathscr{G}_{\gamma}: \gamma<\kappa\right\}$ of open covers of $X$ such that if $x \neq y$, there exists $\gamma<\kappa$ such that $y \notin$ $\operatorname{st}\left(x, \mathscr{G}_{\gamma}\right)$. For $x \in X, \gamma<\kappa$ let $V(\gamma, x)=s t\left(x, \mathscr{G}_{\gamma}\right)$. Then (S) clearly holds, and $e(X) \leq \kappa$ implies that (HC) holds (see (3) of Lemma 1). By Theorem I, $|X| \leq 2^{\kappa}$.

Corollary 3 (Erdös-Rado Partition Theorem, $n=2$ ). Let $\kappa$ be an infinite cardinal, let $E$ be a set with $|E|>2^{\kappa}$, and let $[E]^{2}=\bigcup_{\gamma<\kappa} P_{\gamma}$. Then there exists $\gamma<\kappa$ and $B \subset E$ with $|B|>\kappa$ such that $[B]^{2} \subset P_{\gamma}$.

Proof. For $x \in E$ and $\gamma<\kappa$ let

$$
V(\gamma, x)=\{x\} \cup\left\{y: y \in E, y \neq x \text { and }\{x, y\} \notin P_{\gamma}\right\} .
$$

Since $[E]^{2}=\bigcup_{\gamma<\kappa} P_{\gamma}$, (S) holds. But $|E|>2^{\kappa}$, so by Theorem I there is some subset $A$ of $E$ and some $\gamma<\kappa$ for which (HC) fails. Construct a sequence $B=\left\{x_{\alpha}: 0 \leq \alpha<\kappa^{+}\right\}$of distinct points of $A$ such that for all $\alpha<\kappa^{+}$, $x_{\alpha} \notin \bigcup_{\beta<\alpha} V\left(\gamma, x_{\beta}\right)$. Then $|B|>\kappa$ and $[B]^{2} \subset P_{\gamma}$.

Not surprising is the fact that Theorem I can be obtained from Corollary 3 .

Erdös-Rado Partition Theorem $\Rightarrow$ Theorem I. Let $\{V(\gamma, x): x \in E, \gamma<\kappa\}$ satisfy (S) and (HC), but suppose $|E|>2^{\kappa}$. Let $\leq$ be a well ordering on $E$, and for each $\gamma<\kappa$ let

$$
P_{\gamma}=\{\{x, y\}: x<y \text { and } y \notin V(\gamma, x) \text {, or } y<x\} \text {. }
$$

By (S), $[E]^{2} \subset \bigcup_{\gamma<\kappa} P_{\gamma}$, so there exists $A \subset E$ with $|A|>\kappa$ and $\gamma<\kappa$ such that $[A]^{2} \subset P_{\gamma}$. Choose a subset $A_{0}$ of $A$ with $\left|A_{0}\right|=\kappa^{+}$such that $\left\langle A_{0}, \leq\right\rangle$ is order isomorphic to $\kappa^{+}$(thus no subset of $A_{0}$ of cardinality $\leq \kappa$ runs through $\left.A_{0}\right)$. By (HC), there exists $B \subset A_{0}$ with $|B| \leq \kappa$ such that $A_{0} \subset \bigcup_{x \in B} V(\gamma, x)$. Choose $y \in A_{0}$ such that $x<y$ for all $x \in B$, and then choose $x \in B$ such that $y \in V(\gamma, x)$. Now $\{x, y\} \in P_{\gamma}$ and $x<y$, hence $y \notin V(\gamma, x)$, a contradiction.

Once the Erdös-Rado Partition Theorem is available, there are easy proofs of the Hajnal-Juhász inequalities $|X| \leq 2^{s(X) \psi(X)}$ and $|X| \leq 2^{c(X) \chi(X)}$; see [HJ] or [H3, p. 21]. In fact, these proofs actually yield set-theoretic versions of the two inequalities (Corollaries 4 and 5 below). In the following, PD is for pairwise disjoint, HS is for Hausdorff separating, US is for uniform separating, and D is for discrete. 
Corollary 4. Let $\kappa$ be an infinite cardinal, let $E$ be a set. For each $x \in E$ and each $\gamma<\kappa$ let $V(\gamma, x)$ be a subset of $E$ which contains $x$. Assume that

(HS) if $x \neq y$, there exists $\gamma<\kappa$ such that $V(\gamma, x) \cap V(\gamma, y)=\varnothing$;

(PD) if $\gamma<\kappa$ and $A$ is a subset of $E$ such that $\{V(\gamma, x): x \in A\}$ is pairwise disjoint, then $|A| \leq \kappa$;

then $|E| \leq 2^{\kappa}$.

Corollary 5. Let $\kappa$ be an infinite cardinal, let $E$ be a set. For each $x \in E$ and each $\gamma<\kappa$ let $V(\gamma, x)$ be a subset of $E$ which contains $x$. Assume that

(US) if $x \neq y$, there exists $\gamma<\kappa$ such that $y \notin V(\gamma, x)$ and $x \notin V(\gamma, y)$;

(D) if $\gamma<\kappa$ and $A$ is a subset of $E$ such that for all $x, y$ in $A$ with $x \neq y, y \notin V(\gamma, x)$, then $|A| \leq \kappa$;

then $|E| \leq 2^{\kappa}$.

\section{SeCOND COMBINATORIAL THEOREM}

From Theorem I we obtain a number of the fundamental inequalities in cardinal functions. Obviously missing is the Arhangel'skii inequality $|X| \leq$ $2^{L(X) \chi(X)}$. Theorem II of this section gives a generalization of this important inequality. In the statement of the theorem, HS is for Hausdorff separating, C is for cover.

Theorem II. Let $\kappa$ be an infinite cardinal, let $E$ be a set. For each $x \in E$ and each $\gamma<\kappa$ let $V(\gamma, x)$ be a subset of $E$ which contains $x$. Assume that

(HS) if $x \neq y$, there exists $\gamma<\kappa$ such that $V(\gamma, x) \cap V(\gamma, y)=\varnothing$;

(C) if $\mathscr{V}$ is a subcollection of $\{V(\gamma, x): \gamma<\kappa, x \in E\}$ which covers $E$, there is a subcollection of $\mathscr{V}$ of cardinality $\leq \kappa$ which covers $E$.

Then $|E| \leq 2^{\kappa}$.

Proof. The proof is similar to Pol's proof of Arhangel'skiī's inequality, but we need to replace the closure operation in a topological space. For $L \subset E$ let

$$
L^{*}=\{x: x \in E, V(\gamma, x) \cap L \neq \varnothing \text { for all } \gamma<\kappa\} .
$$

We first prove two properties of this operator.

(P1) if $|L| \leq 2^{\kappa}$, then $\left|L^{*}\right| \leq 2^{\kappa}$;

(P2) if $L=\bigcup_{\alpha<\kappa^{+}} E_{\alpha}^{*}$, where $\left\{E_{\alpha}: 0 \leq \alpha<\kappa^{+}\right\}$is a sequence of subsets of $E$ with $\bigcup_{\beta<\alpha} E_{\beta}^{*} \subset E_{\alpha}$ for all $\alpha<\kappa^{+}$, then $L^{*}=L$.

(P1) is proved by constructing a one-to-one function $\Phi: L^{*} \rightarrow{ }^{\kappa} L$; since $\left|{ }^{\kappa} L\right|=|L|^{\kappa} \leq\left(2^{\kappa}\right)^{\kappa}=2^{\kappa}$, we then have $\left|L^{*}\right| \leq 2^{\kappa}$. For $x \in L^{*}$, let $\Phi(x)=F_{x}$, where $F_{x}$ is a function from $\kappa$ into $L$ such that for all $\gamma<k, F_{x}(\gamma) \in$ $L \cap V(\gamma, x)$. The condition (HS) implies that $\Phi$ is one-to-one.

To prove (P2), it suffices to show that $L^{*} \subset L$. Let $x \in L^{*}$; for each $\gamma<\kappa$ there exists $x_{\gamma} \in V(\gamma, x) \cap L$. Choose $\alpha<\kappa^{+}$so large that $\left\{x_{\gamma}: \gamma<\kappa\right\} \subset$ $\bigcup_{\beta<\alpha} E_{\beta}^{*}$. Now $\bigcup_{\beta<\alpha} E_{\beta}^{*} \subset E_{\alpha}$ and so $V(\gamma, x) \cap E_{\alpha} \neq \varnothing$ for all $\gamma<k$. It fo!lows that $x \in E_{\alpha}^{*}$, hence $x \in L$. 
We now turn to the proof that $|E| \leq 2^{\kappa}$. Construct sequences $\left\{E_{\alpha}: 0 \leq \alpha<\right.$ $\left.\kappa^{+}\right\}$and $\left\{\mathscr{V}_{\alpha}: 1 \leq \alpha<\kappa^{+}\right\}$such that for $\alpha<\kappa^{+}$:

(1) $E_{\alpha}$ is a subset of $E$ with $\left|E_{\alpha}\right| \leq 2^{\kappa}$;

(2) $\bigcup_{\beta<\alpha} E_{\beta}^{*} \subset E_{\alpha}$;

(3) $\mathscr{V}_{\alpha}=\left\{V(\gamma, x): x \in \bigcup_{\beta<\alpha} E_{\beta}^{*}, \gamma<\kappa\right\}$;

(4) if $W$ is the union of at most $\kappa$ elements of $\mathscr{V}_{\alpha}$ and $W \neq E$, then $E_{\alpha}-W \neq \varnothing$.

Let $L=\bigcup_{\alpha<\kappa^{+}} E_{\alpha}^{*}$; then $|L| \leq 2^{\kappa}$, and so the proof is complete if $L=E$. Suppose not, and let $y \in E-L$. For each $x \in L$, choose $\gamma_{x}$ such that $y \notin V\left(\gamma_{x}, x\right)$, and for each $x \notin L\left(=L^{*}\right.$ by (P2)) choose $\gamma_{x}$ such that $V\left(\gamma_{x}, x\right) \cap L=\varnothing$. The collection $\left\{V\left(\gamma_{x}, x\right): x \in E\right\}$ covers $E$, so by (C) there is a subset $B$ of $E$ with $|B| \leq \kappa$ such that $\left\{V\left(\gamma_{x}, x\right): x \in B\right\}$ covers $E$. Let $B_{0}=B \cap L$ and let $W=\bigcup_{x \in B_{0}} V\left(\gamma_{x}, x\right)$. It is clear from the construction of the original cover of $E$ that $L \subset W$ and $y \notin W$. Now choose $\alpha$ so large that $B_{0} \subset \cup_{\beta<\alpha} E_{\beta}^{*}$. By (3) $E_{\alpha}-W \neq \varnothing$, and this contradicts $L \subset W$.

From Theorem II it is possible to obtain a generalization of Arhangel'skiī's inequality. First we define a new cardinal function which we call Hausdorff pseudocharacter and denote $H \psi$. This cardinal function is defined only for Hausdorff spaces, and for such spaces $\psi(X) \leq H \psi(X) \leq \chi(X)$.

Definition. Let $X$ be a Hausdorff space. The Hausdorff pseudo-character of $X$ is the smallest infinite cardinal $\kappa$ such that for every $x \in X$, there is a collection $\mathscr{U}_{x}$ of open neighborhoods of $x$ with $\left|\mathscr{U}_{x}\right| \leq \kappa$ such that if $x \neq y$, there exist $U \in \mathscr{U}_{x}$ and $V \in \mathscr{U}_{y}$ with $U \cap V=\varnothing$.

Corollary 6. For $X \in T_{2},|X| \leq 2^{L(X) H \psi(X)}$.

Proof. Let $L(X) H \psi(X)=\kappa$, and for each $x \in X$ let $\{U(\gamma, x): \gamma<\kappa\}$ be a collection of open neighborhoods of $x$ satisfying the condition for Hausdorff pseudo-character. For $x \in X$ and each pair $\gamma, \delta<\kappa$ let

$$
V(\{\gamma, \delta\}, x)=U(\gamma, x) \cap U(\delta, x) .
$$

Then (HS) and (C) are satisfied and so by Theorem II we have $|X| \leq 2^{\kappa}$.

We note that the Hajnal-Juhász inequality $|X| \leq 2^{c(X) \chi(X)}$ also holds with character weakened to Hausdorff pseudo-character (see $\S 1$, Corollary 4). In other words we have:

$$
|X| \leq 2^{c(X) H \psi(X)} \text {, for } X \in T_{2} .
$$

Example (Sierpiński). There is a Hausdorff, hereditarily Lindelöf space $X$ with $H \psi(X)=\omega$ and $t(X)>\omega$. Let $\mathscr{T}$ denote the usual topology on the set $R$ of real numbers, and let $\mathscr{T}^{*}=\{V-A: V \in \mathscr{T}, A \subset R$ and $A$ countable $\}$. Then $\mathscr{T}^{*}$ is a stronger topology on $R$ and the space $\left(R, \mathscr{T}^{*}\right)$ has the desired properties. 


\section{THIRD COMBINATORIAL THEOREM}

Let $K(X)$ denote the collection of all compact subsets of $X$. Burke and Hodel [BH] have proved that $|K(X)| \leq 2^{e(X) p s w(X)}$. The first step is to show that $|X| \leq 2^{e(X) p s w(X)}$; Miščenko's lemma is then used to finish the proof. Two proofs of the inequality $|X| \leq 2^{e(X) p s w(X)}$ are given in [BH]; one uses an intersection theorem of Erdös-Rado, the other uses the closure method (similar to the proofs of Theorems I and II). As we now show, the two proofs actually yield different bounds which happen to coincide when $\kappa=\lambda$.

In the proof of Theorem III below we use the following result of Engelking and Karlowicz [EK]; its proof depends upon an intersection theorem of ErdösRado.

Lemma. Let $\kappa$ and $\lambda$ be infinite cardinals, let $\left\{A_{t}: t \in T\right\}$ and $\left\{B_{t}: t \in T\right\}$ be collections of sets satisfying these three conditions:

(1) $\left|A_{t}\right| \leq \kappa$ and $\left|B_{t}\right| \leq \lambda$ for all $t \in T$;

(2) $A_{t} \cap B_{t}=\varnothing$ for all $t \in T$;

(3) for $s, t$ distinct elements of $T, A_{s} \cap B_{t} \neq \varnothing$.

Then $|T| \leq \lambda^{\kappa}$.

In the statement of Theorem III, $\mathrm{O}$ is for order and $\mathrm{C}$ is for cover.

Theorem III. Let $\kappa, \lambda$ be infinite cardinals, let $E$ be a set, let $\mathscr{S}$ be a separating cover of $E$. Assume that

(O) for all $x \in E, \operatorname{ord}(x, \mathscr{S}) \leq \kappa$;

(C) if $\mathscr{S}_{0}$ is a subcollection of $\mathscr{S}$ which covers $E$, then some subcollection of $\mathscr{S}_{0}$ of cardinality at most $\lambda$ covers $E$.

Then $|\mathscr{S}| \leq \min \left\{\lambda^{\kappa}, \kappa^{\lambda}\right\}$.

Proof. We first use the closure method to show that $|\mathscr{S}| \leq \kappa^{\lambda}$. Construct a sequence $\left\{E_{\alpha}: 0 \leq \alpha<\lambda^{+}\right\}$of subsets of $E$ and a sequence $\left\{\mathscr{S}_{\alpha}: 1 \leq \alpha<\lambda^{+}\right\}$ of subcollections of $\mathscr{S}$ such that for $\alpha<\lambda^{+}$:

(1) $\left|E_{\alpha}\right| \leq \kappa^{\lambda}$ and $\left|\mathscr{S}_{\alpha}\right| \leq \kappa^{\lambda}$;

(2) $\left.\mathscr{S}_{\alpha}^{\alpha}=\left\{S: S \in \mathscr{S}, S \cap \bigcup_{\beta<\alpha} E_{\beta}\right) \neq \varnothing\right\}$;

(3) if $W$ is the union of at most $\lambda$ elements of $\mathscr{S}_{\alpha}$ and $W \neq E$, then $E_{\alpha}-W \neq \varnothing$.

Let $L=\bigcup_{\alpha<\lambda^{+}} E_{\alpha}$, and note that $|L| \leq \kappa^{\lambda} \cdot \lambda^{+}=\kappa^{\lambda}$. We now argue that every element of $\mathscr{S}^{\alpha}$ intersects $L$. If this is so, it follows from (O) that $|\mathscr{S}| \leq$ $|L| \cdot \kappa \leq \kappa^{\lambda} \cdot \kappa=\kappa^{\lambda}$.

Let $S_{0} \in \mathscr{S}$ but suppose that $S_{0} \cap L=\varnothing$. Let $y \in S_{0}$ and let $\mathscr{G}=\{S: S \in$ $\mathscr{S}, y \notin S\}$. Since $\mathscr{S}$ is separating, $\mathscr{G} \cup\left\{S_{0}\right\}$ is a subcollection of $\mathscr{S}$ which covers $E$. By $(C)$ there is a subcollection $\mathscr{H}$ of $\mathscr{G}$ of cardinality at most $\lambda$ such that $\mathscr{H}$ covers $E-S_{0}$. Let $\mathscr{W}=\{S: S \in \mathscr{H}, S \cap L \neq \varnothing\}$, and note that $|\mathscr{W}| \leq \lambda$ and that $\mathscr{W}$ covers $L$. Choose $\alpha$ so large that $\mathscr{W} \subset \mathscr{S}_{\alpha}$, and 
let $W=\cup \mathscr{W}$. Now $y \notin W$ so $W \neq E$, hence by (3) $E_{\alpha}-W=\varnothing$. This contradicts $L \subset W$.

We now use the Engelking-Karlowicz Theorem to show that $|\mathscr{S}| \leq \lambda^{\kappa}$. For each $x \in E$ let

$$
\mathscr{S}_{x}=\{S: x \in S\}, \quad \mathscr{L}_{x}=\{S: x \notin S\} .
$$

Note that $\left|\mathscr{S}_{x}\right| \leq \kappa$ by (O). Fix $x \in E$ and write $\mathscr{S}_{x}=\left\{S_{\alpha}: 0 \leq \alpha<\kappa\right\}$. For each $\alpha<\kappa,\left\{S_{\alpha}\right\} \cup \mathscr{L}_{x}$ is a subcollection of $\mathscr{S}^{x}$ which covers $E$, so by (C) there exists $\mathscr{W}_{\alpha} \subset \mathscr{L}_{x}$ with $\left|\mathscr{W}_{\alpha}\right| \leq \lambda$ and $\mathscr{W}_{\alpha}$ covers $E-S_{\alpha}$. Moreover, $\mathscr{W}_{x}=\bigcup_{\alpha<\kappa} \mathscr{W}_{\alpha}$ is a subcollection of $\mathscr{S}$ with $\left|\mathscr{W}_{x}\right| \leq \lambda \cdot \kappa$ which covers $E-\{x\}$.

In summary, we have subcollections $\left\{\mathscr{S}_{x}: x \in E\right\}$ and $\left\{\mathscr{W}_{x}: x \in E\right\}$ of $\mathscr{S}$ satisfying

(1) $\left|\mathscr{S}_{x}\right| \leq \kappa$ and $\left|\mathscr{W}_{x}\right| \leq \lambda \cdot \kappa$

(2) $\mathscr{S}_{x} \cap \mathscr{W}_{x}=\varnothing$

(3) for $x \neq y, \mathscr{S}_{y} \cap \mathscr{W}_{x} \neq \varnothing$ (recall that $\mathscr{W}_{x}$ covers $\left.E-\{x\}\right)$.

Thus the Engelking-Karlowicz Theorem applies and $|E| \leq(\lambda \cdot \kappa)^{\kappa}=\lambda^{\kappa}$. It follows that $|\mathscr{S}| \leq \lambda^{\kappa} \cdot \kappa=\lambda^{\kappa}$.

Corollary 7 (Charlesworth). For $X \in T_{1}, n w(X) \leq p s w(X)^{L(X)}$.

Proof. Let psw $(X)=\kappa, L(X)=\lambda$, and $\mathscr{S}$ be a separating open cover of $X$ with $\operatorname{ord}(x, \mathscr{S}) \leq \kappa$ for all $x \in X$. By Theorem III we have $|\mathscr{S}| \leq \kappa^{\lambda}$. Let $\mathscr{N}=\{X-S: S$ is the union of at most $\lambda$ elements of $\mathscr{S}\}$. Then $|\mathcal{N}| \leq\left(\kappa^{\lambda}\right)^{\lambda}=\kappa^{\lambda}$ and $\mathscr{N}$ is a net for $X$.

Before stating the next application of Theorem III we give a generalization of extent. For any space $X$ the weak extent of $X$ is the smallest infinite cardinal $\kappa$ such that if $\mathscr{G}$ is any open cover of $X$, there is a subset $B$ of $X$ with $|B| \leq \kappa$ such that $\operatorname{st}(B, \mathscr{G})=X$. It is clear from Lemma 1 that $w e(X) \leq e(X)$; note also that $w e(X) \leq d(X)$. This cardinal function was first introduced by MuMing in [MM]; he calls this cardinal function the ${ }^{*}$ Lindelöf number and denotes it by ${ }^{*} L(X)$. MuMing and Sun-Wang [SW] independently proved that $|K(X)| \leq 2^{w e(X) p s w(X)}$, thereby generalizing the Burke-Hodel inequality. Corollary 8 generalizes the MuMing-Sun-Wang inequality.

Corollary 8. For $X \in T_{1},|K(X)| \leq w e(X)^{p s w(X)}$.

Proof. Let $p s w(X)=\kappa, w e(X)=\lambda$, and let $\mathscr{S}$ be a separating open cover of $X$ such that $\operatorname{ord}(x, \mathscr{S}) \leq \kappa$ for all $x \in X$. As a first step, we use Theorem III to prove that $|\mathscr{S}| \leq \lambda^{\kappa}$. To check (C), let $\mathscr{S}_{0}$ be a subcollection of $\mathscr{S}$ which covers $X$. Since $w e(X)=\lambda$, there exists $B \subset X$ with $|B| \leq \lambda$ such that $\operatorname{st}\left(B, \mathscr{S}_{0}\right)=X$. Now ord $(x, \mathscr{S}) \leq \kappa$ for all $x \in X$, and thus $\left\{S \in \mathscr{S}_{0}\right.$ : $S \cap B \neq \varnothing\}$ is a subcollection of $\mathscr{S}_{0}$ of cardinality at most $\kappa \cdot \lambda$ which covers $X$. By Theorem III we conclude that $|\mathscr{S}| \leq(\kappa \cdot \lambda)^{\kappa}=\lambda^{\kappa}$.

Next we prove that $|X| \leq \lambda^{\kappa}$. Let $\mathscr{S}^{*}=\{S: S$ is the intersection of at most $\kappa$ elements of $\mathscr{S}\}$. Now $\left|\mathscr{S}^{*}\right| \leq \lambda^{\kappa}$, and the function $\Phi: X \rightarrow \mathscr{S}^{*}$ defined 
by $\Phi(x)=\cap\{S: x \in S\}$ is one-to-one ( $\mathscr{S}$ is separating), hence $|X| \leq \lambda^{\kappa}$. Finally, one can use Miščenko's Lemma to show that $|K(X)| \leq \lambda^{\kappa}$; see $[\mathrm{BH}]$ for details.

The inequality in Corollary 8 is new. To illustrate, suppose $X$ is a $T_{1}$ space with a point-countable separating open cover such that every closed, discrete subset of $X$ has cardinality at most $2^{\omega}$. Then the number of compact subsets of $X$ is at most $2^{\omega}$.

Concluding remarks. The closure method, also referred to as the Pol-Šapirovskii technique in [H2] and [H3] (see [P, S]), is fundamental in the proofs of Theorems I, II, and III. This is obvious in the case of the first two theorems and the first part of the third theorem. Moreover Michael [M] has given a proof of the Erdös-Rado Intersection Theorem using the closure method.

\section{REFERENCES}

[A] A. V. Arhangel' skii, On the cardinality of bicompacta satisfying the axiom of first countability, Soviet Math. Dokl. 10 (1969), 951-955.

[BH] D. K. Burke and R. E. Hodel, The number of compact subsets of a topological space, Proc. Amer. Math. Soc. 58 (1976), 363-368.

[C] A. Charlesworth, On the cardinality of a topological space, Proc. Amer. Math. Soc. 66 (1977), 138-142.

[E] R. Engelking, General topology, Heldermann, Berlin, 1989.

[EK] R. Engelking and M. Karlowicz, Some theorems of set theory and their topological consequences, Fund. Math. 57 (1965), 275-285.

[ER1] P. Erdös and R. Rado, A partition calculus in set theory, Bull. Amer. Math. Soc. 62 (1956), 427-489.

[ER2] _ Intersection theorems for systems of sets, J. London Math. Soc. 35 (1960), 85-90.

[GW] J. Ginsburg and G. Woods, A cardinal inequality for topological spaces involving closed discrete sets, Proc. Amer. Math. Soc. 64 (1977), 357-360.

[G] J. de Groot, Discrete subspaces of Hausdorff spaces, Bull. Acad. Polon. Sci. 13 (1965), 537544.

[HJ] A. Hajnal and I. Juhász, Discrete subsets of topological spaces, Indag. Math. 29 (1967), 343-356.

[H1] R. Hodel, On a theorem of Arhangel' skii concerning Lindelöf p-spaces, Canad. J. Math. 27 (1975), 459-468.

[H2] _ A technique for proving inequalities in cardinal functions, Topology Proc. 4 (1979), 115-120.

[H3] _ Cardinal functions. I, in Handbook of Set-Theoretic Topology (K. Kunen and J. E. Vaughan, eds.), North-Holland, Amsterdam, 1984, pp. 1-61.

[J] I. Juhász, Cardinal functions in topology-ten years later, Mathematisch Centrum, Amsterdam, 1980.

[M] E. Michael, A note on intersections, Proc. Amer. Math. Soc. 13 (1962), 281-283.

[Mi] A. Miščenko, Spaces with point-countable bases, Soviet Math. Dokl. 3 (1962), 855-858.

[MM] Dai MuMing, A topological space cardinality inequality involving the ${ }^{*}$ Lindelöf number, Acta Math. Sinica 26 (1983), 731-735.

[P] R. Pol, Short proofs of two theorems on cardinality of topological spaces, Bull. Acad. Polon. Sci. 22 (1974), 1245-1249. 
[S] B. Šapirovskiī, On discrete subspaces of topological spaces. Weight, tightness and Souslin number, Soviet Math. Dokl. 13 (1972), 215-219.

[SW] S. H. Sun and Y. M. Wang, A strengthened topological cardinal inequality, Bull. Austral. Math. Soc. 32 (1985), 375-378.

Department of Mathematics, Duke University, Durham, North Carolina 27706 